\title{
Planar nanowire arrays formed by atomic-terrace low-angle shadowing
}

\author{
F. Cuccureddu, V. Usov, ${ }^{\text {a) }}$ S. Murphy, C. O. Coileain, and I. V. Shvets \\ Centre for Research on Adaptive Nanostructures and Nanodevices (CRANN), School of Physics, \\ Trinity College, Dublin 2, Ireland
}

(Received 25 March 2008; accepted 28 April 2008; published online 20 May 2008)

\begin{abstract}
A relatively simple method for preparation of planar nanowire arrays on vicinal substrates by molecular beam epitaxy is presented. The atomic step-and-terrace morphology of vicinal substrates is used to produce a shadowing effect on a highly collimated molecular beam at an oblique incidence to the substrate. The collimation is achieved by placing the evaporation source at a large working distance $(40-100 \mathrm{~cm})$ from the substrate. The method's capabilities have been demonstrated by preparation of arrays of $\mathrm{Ag}$ and $\mathrm{Au}$ nanowires on vicinal $\mathrm{Si}(111)$ and $\alpha-\mathrm{Al}_{2} \mathrm{O}_{3}$ (0001) substrates. Nanowires with a width of down to $10-15 \mathrm{~nm}$ and a thickness of $1.5 \mathrm{~nm}$ have been readily achieved. (C) 2008 American Institute of Physics. [DOI: 10.1063/1.2929835]
\end{abstract}

The bottom-up approach to the fabrication of nanostructures has become a popular subject in current science and engineering. In particular, there has been substantial interest in the fabrication of nanowires of many different materials, driven by a wide range of potential applications. These nanowires come in many shapes and sizes but for nanowires formed by molecular beam epitaxy (MBE) on a substrate we can identify two principal geometries, i.e., either out of the substrate surface, ${ }^{1,2}$ or in plane with the surface, e.g., planar nanowires grown on vicinal surfaces by the step-flow growth mechanism. ${ }^{3,4}$ The latter are particularly interesting from the point of view of planar electronic applications. However, preparation of nanowires by the step-flow growth mechanism is restricted in that it only works well for certain materials on certain metal or semiconductor surfaces. This is unfortunate if one is interested, for example, in utilizing the insulating properties of an oxide substrate. To overcome this difficulty, we look on to utilizing the shadowing effect of the step-andterrace morphology of a vicinal surface on a molecular beam at oblique incidence to the substrate surface. We term this approach atomic-terrace low-angle shadowing (ATLAS). This method utilizes the advantages of the bottom-up fabrication and its principal attraction is its relative simplicity. The method does not involve multiple lithography steps and can be applied to metal, semiconductor, or oxide surfaces alike, thus, making it potentially interesting for practical applications. ${ }^{5}$ Vicinal surfaces can be formed by annealing a surface that is off cut from a low-index orientation. ${ }^{6-8}$ It is composed of atomic terraces separated by steps. The width of the atomic terraces can be readily controlled by the off-cut angle. Therefore, the separation between the nanowires within the array and their width could be readily controlled.

The schematic of the ATLAS technique is shown in Fig. 1. In Fig. 1(a), a collimated beam of evaporant atoms is directed from an outer step edge to a lower terrace (i.e., a descending step direction). The deposition angle $\beta$ between the beam and the surface is only $1-3^{\circ}$ in order that some areas of the atomic terraces are exposed to the beam, while

\footnotetext{
a) Author to whom correspondence should be addressed. Electronic mail: usovv@tcd.ie.
}

others are geometrically shadowed by atomic steps. As a result, the deposited material grows along the terrace step edges. Changing the deposition angle $\beta$ changes the nanowire width which is given by

$$
w \approx a\left(\frac{1}{\alpha}-\frac{1}{\alpha+\beta}\right),
$$

where $w$ is a nanowire width, $a$ is a step height, $\alpha$ is a miscut angle, and $\beta$ is a deposition angle, defined as the angle between the surface and the incident beam [Fig. 1(a)]. Both $\alpha$ and $\beta$ are in radians and assumed to be small.

In Fig. 1(b), the substrate is rotated by $180^{\circ}$ and the beam is incident nearly parallel to the atomic terraces (i.e., an ascending step direction). The step faces provide the bonding sites and the deposited material grows along the steps similar to the step-flow growth mechanism. In order to grow wires with the width of fifteen nanometers or less, the deposition temperature should be chosen for both orientations such that the adatom free path on the substrate is limited to a few nanometers.

A highly collimated molecular beam is achieved, in our experiments, by using a large working distance between the substrate and the evaporation source. Our deposition system
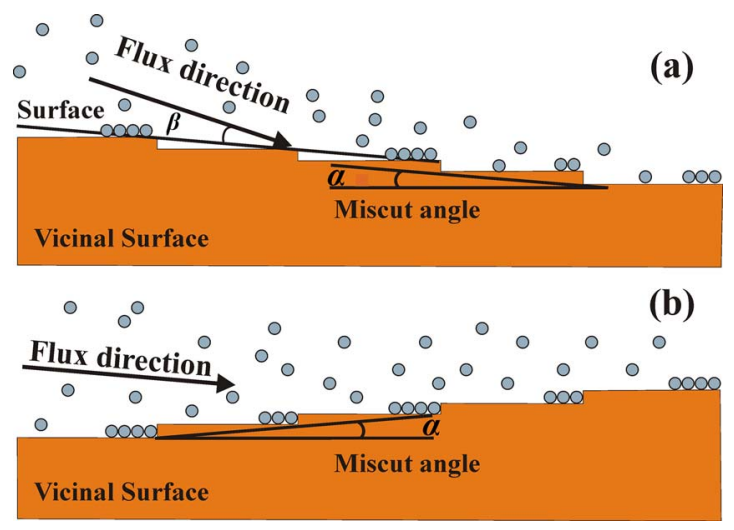

FIG. 1. (Color online) Schematics of deposition at a shallow angle on a vicinal surface (a) deposition of nanowires on the outer step edges; the beam is directed at an angle $\beta$ along the descending step direction (b) the substrate is rotated by $180^{\circ}$ and the beam is directed in the ascending step direction. 


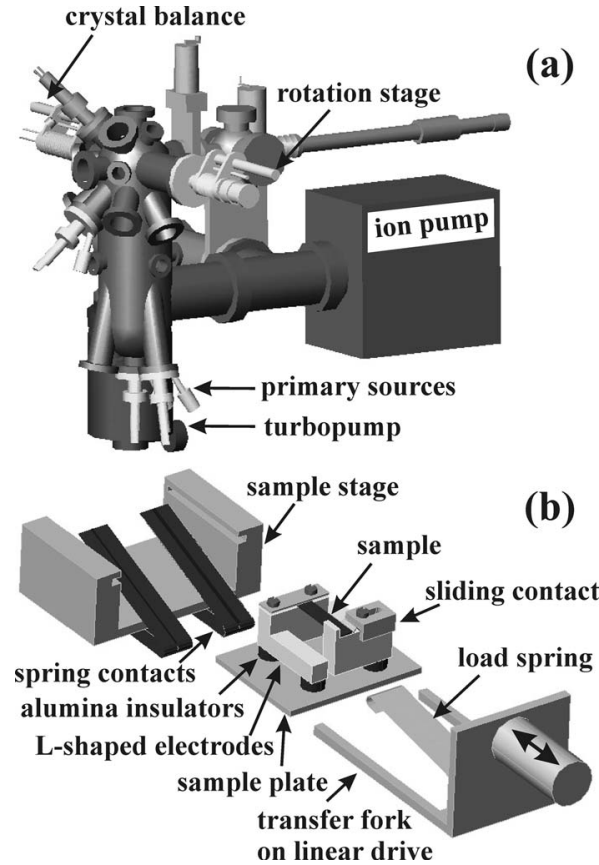

FIG. 2. (a) Schematic illustration of the ATLAS vacuum system (b) Sample stage and transfer.

[Fig. 2(a)] is a $200 \mathrm{~mm}$ diameter stainless steel ultrahigh vacuum (UHV) chamber equipped with a fast-entry loadlock. Typical base pressure achieved in this system is in the low $10^{-10}$ Torr range. The sample is mounted at the main focal point of the chamber and can be tilted through $200^{\circ}$ with a precision of $\pm 0.5^{\circ}$. This allows for both sample transfer and alignment relative to the primary and secondary sources. The primary source is either a $10 \mathrm{cc}$ hightemperature effusion cell or an electron beam evaporator (both MBE-Komponenten). The effusion cell is used to evaporate metals while the e-beam evaporator together with a $P$-doping source is used to grow $\mathrm{Si}$ nanowires. The minimum working distance between the primary source and a substrate is $370 \mathrm{~mm}$. This is normally extended to $800 \mathrm{~mm}$ by mounting the source on a straight connector. One or more secondary sources can be mounted at the front of the chamber to allow for the normal deposition of passivating or functional layers over the nanowire arrays.

The substrate is mounted onto two Ta L-shaped electrodes [Fig. 2(b)]. One side of the substrate is clamped while the other is secured with a spring-loaded sliding contact that leaves the substrate open to the plume of deposited material. The electrodes are connected to a stainless steel sample plate by electrically isolated screws. The screws are pressed against two spring contacts which are mounted on a sample stage and connected to a power supply via feedthroughs. The semiconducting samples are heated by dc while the insulating oxide substrates are heated by driving the current through a Ta foil which is placed underneath the substrate.

The sample stage is machined with the inner grooves to accept the sample plate. The sample plate with a mounted sample is docked by a spring-loaded transfer fork at the end of a linear drive. It is transferred into the vacuum chamber and docked with the stage by sliding the plate into the grooves until the screws on a plate are pressed against the spring contacts. The fork's load spring is made in a hooklike shape so that is can "catch" the sample plate so that it may be pulled out of the sample stage afterwards and removed from the chamber.

The capabilities of the ATLAS method were demonstrated by forming arrays of $10-30 \mathrm{~nm}$ wide metallic nanowires on two types of vicinal substrates: $\mathrm{Si}(111)$ and $\alpha-\mathrm{Al}_{2} \mathrm{O}_{3}$ (0001) ( $c$ plane of sapphire). $\mathrm{Ag}$ and $\mathrm{Au}$ nanowires were grown on $\mathrm{Si}(111)$ step-bunched surface at room temperature. The substrates were prepared from a $\mathrm{Si}(111) n$-type doped wafer with a $3^{\circ}$ miscut along the $[-1-12]$ direction. The wafer was cut into $20 \times 4 \times 0.5 \mathrm{~mm}^{3}$ rectangular pieces with the long side along the direction of the miscut. First, the samples were outgassed in UHV for $3 \mathrm{~h}$ at $750{ }^{\circ} \mathrm{C}$ (measured with an infrared pyrometer) and flash annealed for 20-30 times to $1250{ }^{\circ} \mathrm{C}$ for $10-12$ s to obtain a clean surface. Subsequently, the samples were annealed for $3 \mathrm{~h}$ at $920{ }^{\circ} \mathrm{C}$ by passing a dc in the [11-2] (the ascending step) direction. ${ }^{6}$ The obtained surface morphology is characterized by a staircase arrangement of high density terraces which are 20-30 nm wide and separated by $2-2.5 \mathrm{~nm}$ high silicon step bunches [inset of Fig. 3(b)]. A collimated Ag or Au flux was oriented in the descending step direction at $1.2^{\circ}$ to the sample surface.

Prior to growing the nanowires, the deposition angle was calibrated by using a $\mathrm{Si}(111)$ substrate with an identical miscut but with the step bunches of larger dimension. The surface was composed of 180-240 nm high step bunches separated by $1.5-2 \mu \mathrm{m}$ wide $\mathrm{Si}(111)$ terraces. This surface was prepared under the same conditions as previous silicon substrates with the notable exception of being annealed at $1130{ }^{\circ} \mathrm{C} .{ }^{6} 10 \mathrm{~nm}$ of $\mathrm{Ag}$ was deposited on these terraces to produce microscale wires, which provided greater contrast between $\mathrm{Ag}$ and $\mathrm{Si}$ surfaces during atomic force microscopy (AFM) measurements. This allowed the angle of deposition $\beta$, to be accurately measured [inset of Fig. 3(a)].

Figures 3(b) and 3(d) show the AFM images of Ag and $\mathrm{Au}$ nanowires. The nanowire average height, width, and separation are 2,15 , and $30 \mathrm{~nm}$ respectively. It is interesting that the height of the nanowires, some $2 \mathrm{~nm}$, is well in excess of a single atomic layer. The width of $15 \mathrm{~nm}$ is approximately a fivefold reduction in size compared to existing benchmark results for nanowire arrays produced by deposition at a shallow angle.

In another series of experiments, arrays of $\mathrm{Ag}$ nanowires were grown on vicinal $\alpha-\mathrm{Al}_{2} \mathrm{O}_{3}$ substrates which were miscut by $3^{\circ}$ off the $(0001)$ plane along the [1 $\left.\overline{2} 10\right]$ direction. The substrates were annealed in air at $1100{ }^{\circ} \mathrm{C}$ for $6-12 \mathrm{~h}$ to produce a stepped surface. ${ }^{7,8}$ The surface topography, which is shown in Fig. 4(a), is characterized by a staircase arrangement of 50-55 nm wide terraces separated by $1.5-2 \mathrm{~nm}$ high step bunches [Fig. 4(a)]. 11 nanometers of Ag was deposited on this surface at room temperature at a deposition rate of $3.6 \AA /$ min, as measured with a quartz crystal balance, which was positioned normal to the incident flux. The deposition flux was directed to the substrate in the ascending step direction. Atomic steps provide numerous high coordination nucleation sites and reduce the adatom diffusion length. As a result, chains of $\mathrm{Ag}$ islands with an average width of $15-20 \mathrm{~nm}$ and thickness of 1.5-2 $\mathrm{nm}$ formed along the substrate steps, as shown in Figs. 3(b). The size of nanoparticles and spacing between them was changed by depositing 


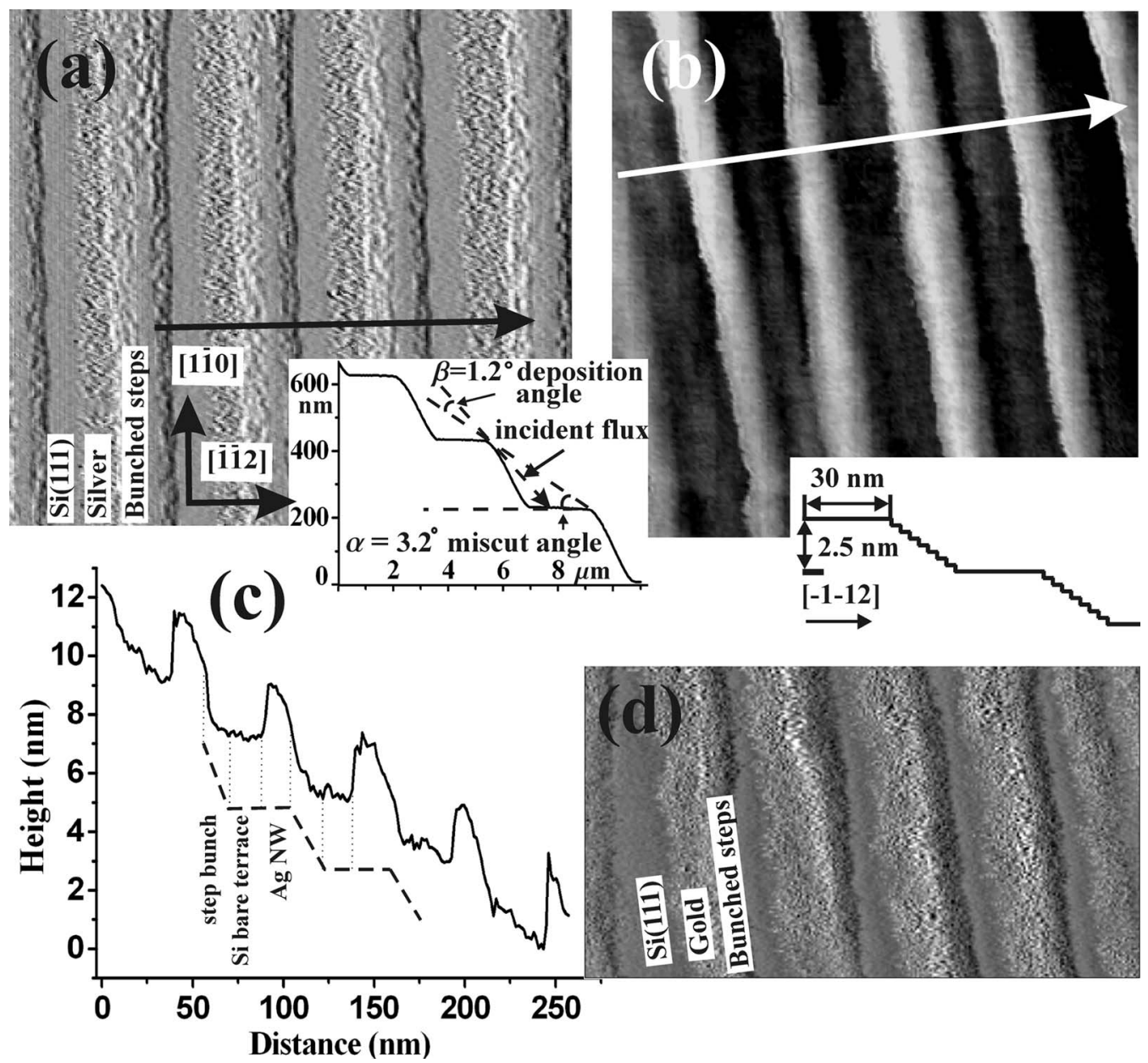

FIG. 3. Planar arrays of nanowires grown by ATLAS technique. (a) $15 \times 13.5 \mu \mathrm{m}^{2}$ feedback error AFM image of Ag wires deposited on a bunched Si(111) surface in order to measure the deposition angle $\beta$; an inset shows the deposition angle measured from a line profile along the miscut direction indicated by an arrow in (a). (b) $250 \times 250 \mathrm{~nm}^{2} \mathrm{AFM}$ image of a planar array of $15 \mathrm{~nm}$ wide Ag nanowires grown at $\beta=1.2^{\circ}$; the inset is a schematic of a $\mathrm{Si}(111)$ substrate along the miscut direction. (c) A line profile along the direction of a miscut marked in (b) and a schematic of an underlying $\mathrm{Si}(111)$ bunched substrate; (d) $300 \times 170 \mathrm{~nm}^{2}$ feedback error AFM image of a planar array of $15 \mathrm{~nm}$ wide Au nanowires grown at $\beta=1.2^{\circ}$.

smaller amount of material. Figure 3(c) shows chains of $12-16 \mathrm{~nm}$ diameter nanoparticles separated by $2-5 \mathrm{~nm}$, which were formed by depositing $6 \mathrm{~nm}$ of $\mathrm{Ag}$ at room temperature at the same deposition rate.

We have described a MBE technique, which allows the growth of nanowires utilizing the atomic step-and-terrace

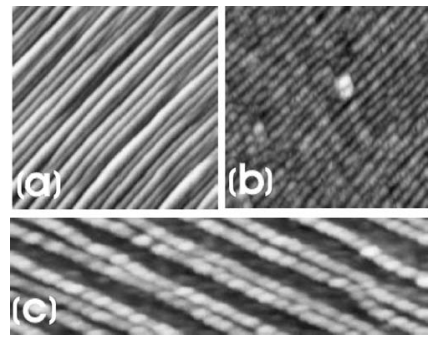

FIG. 4. Chains of $\mathrm{Ag}$ nanoparticles grown on an $\alpha-\mathrm{Al}_{2} \mathrm{O}_{3}(0001)$ vicinal surface. (a) $800 \times 800 \mathrm{~nm}^{2}$ AFM image of a vicinal $\alpha$ - $\mathrm{Al}_{2} \mathrm{O}_{3}(0001)$ surface after annealing in the air for $12 \mathrm{~h}$ at $1100{ }^{\circ} \mathrm{C}$. (b) $780 \times 780 \mathrm{~nm}^{2} \mathrm{AFM}$ image of chains of $15-20 \mathrm{~nm} \mathrm{Ag}$ nanoparticles on an $\alpha-\mathrm{Al}_{2} \mathrm{O}_{3}(0001)$ surface after deposition of $11 \mathrm{~nm}$ of $\mathrm{Ag}$ at room temperature. The deposition flux was directed in the ascending step direction. (c) $510 \times 140 \mathrm{~nm}^{2}$ AFM image of an annealed vicinal $\alpha-\mathrm{Al}_{2} \mathrm{O}_{3}(0001)$ surface with $6 \mathrm{~nm}$ of $\mathrm{Ag}$ deposited at room temperature. The Ag nanoparticles are separated by $2-5 \mathrm{~nm}$ and their lateral size reduced to $12-16 \mathrm{~nm}$. morphology of vicinal substrates. An atomic step shadows a part of an underlying terrace from a collimated evaporant beam so that the atoms form a nanowire on an open area along the step edges. Otherwise, the atoms are directed nearly parallel to the terraces so that the step faces remain exposed to the evaporant beam and the deposited material grows along the steps similar to the step-flow growth mechanism. We have described a UHV setup where a collimated incident beam was achieved by placing a substrate at a large distance from the evaporation source $(40-100 \mathrm{~cm})$. The capabilities of the system have been demonstrated by growing 10-15 nm wide $\mathrm{Au}$ and $\mathrm{Ag}$ nanowires on step-bunched vicinal $\mathrm{Si}(111)$ and $\alpha-\mathrm{Al}_{2} \mathrm{O}_{3}$ (0001) substrates.

The financial support of the Science Foundation Ireland, Contract No. 06/IN.1/191 is gratefully acknowledged. The authors would like to thank the workshop technicians Patrick Murphy and Gordon O'Brian for their assistance in manufacturing components.

${ }^{1}$ V. G. Dubrovskii, G. E. Cirlin, I. P. Soshnikov, A. A. Tonkikh, N. V. Sibirev, Yu. B. Samsonenko, and V. M. Ustinov, Phys. Rev. B 71, 205325 (2005).

${ }^{2}$ N. J. Quitoriano and T. I. Kamins, J. Appl. Phys. 102, 044311 (2007).

${ }^{3}$ T. Jung, R. Schlittler, J. K. Gimzewski, and F. J. Himpsel, Appl. Phys. A: 
Mater. Sci. Process. 61, 467 (1995)

${ }^{4}$ A. Cazacu, S. Murphy, and I. V. Shvets, Phys. Rev. B 74, 045413 (2006).

${ }^{5}$ I. V. Shvets, H. C. Wu, V. Usov, F. Cuccureddu, S. K. Arora, and S.

Murphy, Appl. Phys. Lett. 92, 023107 (2008).

${ }^{6}$ J. L. Lin, D. Y. Petrovykh, J. Viernow, F. K. Men, D. J. Seo, and F. J.
Himpsel, J. Appl. Phys. 84, 255 (1998)

${ }^{7}$ L. Pham Van, O. Kurnosikov, and J. Cousty, Surf. Sci. 411, 263 (1998).

${ }^{8}$ O. Kurnosikov, L. Pham Van, and J. Cousty, Surf. Sci. 459, 256 (2000).

${ }^{9}$ J. Oster, M. Kallmayer, L. Wiehl, H. J. Elmers, H. Adrian, F. Porrati, and

M. Huth, J. Appl. Phys. 97, 014303 (2005). 